\title{
Artificial optical emissions at HAARP for pump frequencies near the third and second electron gyro-harmonic
}

\author{
M. J. Kosch ${ }^{1,2,3}$, T. Pedersen ${ }^{1}$, J. Hughes ${ }^{4}$, R. Marshall ${ }^{5}$, E. Gerken ${ }^{6}$, A. Senior ${ }^{2}$, D. Sentman ${ }^{4}$, M. McCarrick ${ }^{7}$, and \\ F. T. Djuth ${ }^{8}$ \\ ${ }^{1}$ Space Vehicles Directorate, Air Force Research Laboratory, Hanscom AFB, Massachusetts, USA \\ ${ }^{2}$ Communication Systems, Lancaster University, Lancaster, UK \\ ${ }^{3}$ Honorary Research Fellow, University of Kwazulu-Natal, Durban, South Africa \\ ${ }^{4}$ Geophysical Institute, University of Alaska, Fairbanks, USA \\ ${ }^{5}$ STARLab, Stanford University, Stanford CA, USA \\ ${ }^{6}$ Dept. Electrical \& Computer Engineering, Cornell University, Ithaca NY, USA \\ ${ }^{7}$ Advanced Power Technologies, Washington D.C., USA \\ ${ }^{8}$ Geospace Research, El Segundo CA, USA
}

Received: 23 November 2004 - Revised: 23 February 2005 - Accepted: 7 March 2005 - Published: 27 July 2005

Part of Special Issue "Atmospheric studies by optical methods"

\begin{abstract}
High-power high-frequency radio waves beamed into the ionosphere cause plasma turbulence, which can accelerate electrons. These electrons collide with the F-layer neutral oxygen causing artificial optical emissions identical to natural aurora. Pumping at electron gyro-harmonic frequencies has special significance as many phenomena change their character. In particular, artificial optical emissions become strongly reduced for the third and higher gyroharmonics. The High frequency Active Auroral Research Program (HAARP) facility is unique in that it can select a frequency near the second gyro-harmonic. On 25 February 2004, HAARP was operated near the third and passed through the second gyro-harmonic for the first time in a weakening ionosphere. Two novel observations are: firstly, a strong enhancement of the artificial optical emission intensity near the second gyro-harmonic, which is opposite to higher gyro-harmonics; secondly, the optical enhancement maximum occurs for frequencies just above the second gyro-harmonic. We provide the first experimental evidence for these effects, which have been predicted theoretically. In addition, irregular optical structures were created when the pump frequency was above the ionospheric critical frequency.
\end{abstract}

Keywords. Active experiments - Auroral ionosphere Wave-particle interactions

Correspondence to: M. Kosch

(m.kosch@lancaster.ac.uk)

\section{Introduction}

A major goal for active experiments in the ionosphere is to understand the fundamental physics governing the interaction between plasma and electromagnetic waves. High power high frequency pumping of the ionosphere causes several well-known phenomena. These include magnetic field-aligned density irregularities (Robinson et al., 1989; Frolov et al., 1997) of small scale size (1-10 m) (Kelley et al., 1995) called striations, stimulated electromagnetic emissions (Leyser, 2001), anomalous (non-collisional) absorption of low power diagnostic radio waves when they propagate through the pumped volume (Stubbe, 1996), electron temperature enhancements (Stocker et al., 1992; Rietveld et al. 2003) and artificial optical emissions, which are produced by electron collisions with the neutrals. This last phenomenon is now well-established at auroral latitudes at EISCAT in northern Scandinavia (Brändström et al., 1999) and at HAARP in Alaska (Pedersen and Carlson, 2001). Observing these photons is the best means of remotely detecting the energetic electrons, which result from turbulent plasma processes brought on by the pump wave. The energisation threshold for the $\mathrm{O}\left({ }^{1} \mathrm{D}\right) 630 \mathrm{~nm}$ and $\mathrm{O}\left({ }^{1} \mathrm{~S}\right) 557.7 \mathrm{~nm}$ emissions are 1.96 and $4.17 \mathrm{eV}$, respectively, although the effective threshold for $\mathrm{O}\left({ }^{1} \mathrm{D}\right)$ is $\sim 3.5 \mathrm{eV}$ due to collisional quenching by $\mathrm{N}_{2}$ (Haslett and Megill, 1974).

The mechanism for electron acceleration is thought to be electrostatic waves. The two main candidate mechanisms are upper-hybrid waves and Langmuir waves, which act perpendicular and parallel to the magnetic field line, respectively. For high latitudes, Leyser et al. (2000) suggested 
that upper-hybrid turbulence is the dominant mechanism, for which theoretical support (Istomin and Leyser, 2003) and experimental evidence (Kosch et al., 2002a) exists. Upperhybrid waves are generated by resonant mode conversion of the pump electromagnetic wave on plasma irregularities (Robinson, 1989), i.e. striations. This occurs efficiently at the upper-hybrid resonance altitude, which is typically 3$8 \mathrm{~km}$ below the high frequency reflection altitude in the ionosphere. Upper-hybrid waves have a symbiotic relationship with pump-enhanced striations (Dysthe et al., 1982), which are a common feature of ionospheric pumping (e.g. Robinson et al., 1998; Bond et al., 1997) and are associated with artificial optical emissions (Kosch et al., 2002a,b). The growth of striations is due to thermal parametric instabilities, which are stimulated by the pump wave (Fejer, 1979; Robinson, 1989; Mjølhus, 1990). Coherent radar backscatter is produced by Bragg scattering of the sounding waves from either natural or pump-enhanced striations (Stubbe, 1996). Hence, pumpinduced coherent backscatter acts as a proxy for detecting artificial upper-hybrid waves and is routinely observed by the SuperDARN radars (e.g. CUTLASS for EISCAT and Kodiak for HAARP). However, backscatter is only observed when the radar ray is essentially orthogonal to the striations at the point of reflection (Fialer, 1974; Minkoff et al., 1974), hence propagation conditions between the radar and the striations are critical to successful observations (Senior et al., 2004).

Pump wave conversion to Langmuir electrostatic waves is accompanied by the simultaneous production of ion-acoustic waves (Robinson, 1989, 1997) to which incoherent backscatter radars (e.g. EISCAT) are sensitive. Observations of enhanced ion-line (ion-acoustic waves) and plasma-line (Langmuir waves) backscatter just below the pump wave reflection altitude show that Langmuir turbulence is important for the initial few seconds after a pump wave is switched on (e.g. Honary et al., 1999) before the pump-enhanced striations fully establish themselves. In addition, Honary et al. (1999) have shown that Langmuir turbulence persists when pumping on an electron gyro-harmonic frequency because the growth of striations is suppressed (e.g. Ponomarenko et al., 1999; Kosch et al., 2002a). Hence, the high frequency pump wave can reach the higher Langmuir matching height without being dissipated at a lower altitude by mode conversion into upper-hybrid waves (Gurevich 1978; Dysthe et al., 1982). Artificial optical emissions associated with Langmuir turbulence on the fourth gyro-harmonic have been observed (Kosch et al., 2004).

Most ionospheric modification effects are sensitive to the pump frequency relative to the electron gyro-harmonic frequency. Stimulated electromagnetic emissions change their character (Stubbe et al., 1994; Honary et al., 1995; Frolov et al., 2001), anomalous absorption minimizes (Stocker et al., 1993; Stubbe et al., 1994), electron temperature enhancements minimize (Honary et al., 1995; Robinson et al., 1996) and artificial optical emissions minimize (Kosch et al., 2002a; Gustavsson et al., 2004) (with the notable exception mentioned earlier (Kosch et al., 2004)). All these phenomena are linked to striations, and by association upper- hybrid waves, whose growth are suppressed when pumping on a gyro-harmonic frequency (Honary et al., 1995; Robinson et al., 1996). In addition, Istomin and Leyser (2003) predicted theoretically that upper-hybrid waves and striations, and therefore also artificial optical emissions, would be stronger for pump frequencies just above a gyro-harmonic frequency compared to below it, i.e. an asymmetry would exist. A significant caveat with all of the above gyro-harmonic results is that they are only for pumping frequencies well above the second gyro-harmonic.

Until recently, opportunities to perform ionospheric pump experiments on the second gyro-harmonic have been very limited. In the early 1980s, the EISCAT pump facility could operate around the second gyro-harmonic $(2.76 \mathrm{MHz})$ (e.g. Frey, 1986), but no optical or electron temperature data have been published. In the 1970s, experiments using the midlatitude Platteville heater in Colorado, USA, were performed at the fixed pump frequency of $2.85 \mathrm{MHz}$. They produced coherent radar backscatter (Fialer, 1974; Minkoff et al., 1974), i.e. striations and upper-hybrid waves, and artificial optical emissions $(<100$ Rayleigh at $630 \mathrm{~nm}$ ) (Sipler and Biondi, 1972; Haslett and Megill, 1974). When the pump frequency happened to be close to the second gyro-harmonic, exceptionally bright artificial optical emissions were produced (>250 Rayleigh at $630 \mathrm{~nm}$ ) (Haslett and Megill, 1974). One of us (Sentman, D., 2002 campaign) has observed bright artificial optical emissions from the HIPAS facility in Alaska, which also operates at $2.85 \mathrm{MHz}$. Fialer (1974) also noted that coherent backscatter was enhanced when pumping close to the second gyro-harmonic. Theory predicts that the threshold for the thermal parametric instability, which is a mechanism for the growth of striations (Fejer, 1979; Robinson, 1989; Mjølhus, 1990), is reduced close to the second gyroharmonic (Grach, 1979) by as much as a factor of 5-10 (Das and Fejer, 1979). This is only true for an inhomogeneous plasma where the plasma density and magnetic field gradients are anti-parallel (Grach, 1979), which is exactly the situation in the ionosphere below the F-region peak. Das and Fejer (1979) suggest that the reduction in threshold would also result in enhanced striations, which is consistent with the observations (Fialer, 1974; Haslett and Megill, 1974). In addition, Fialer (1974) noted that coherent backscatter power, i.e. striations, maximized when pumping just above the second gyro-harmonic, which is consistent with a modern theory of cylindrical upper-hybrid waves trapped in striations (Istomin and Leyser, 2003), although this theory does not explicitly deal with the second gyro-harmonic. Optical evidence of this effect is presented in this paper for the first time.

\section{Results and analysis}

The High frequency Active Auroral Research Program (HAARP) facility at Gakona $\left(62.4^{\circ} \mathrm{N}, 145.15^{\circ} \mathrm{W}\right)$, Alaska, can operate from $2-10 \mathrm{MHz}$ and therefore covers all gyroharmonics from 2 to 7 . HAARP is currently the only ionospheric heater capable of selecting a pump frequency 
suitable for passing through the second gyro-harmonic of almost any nighttime ionosphere. On 25 February 2004 an experiment was performed, which attempted to pass through the third and second gyro-harmonic frequencies for Kodiak radar (SuperDARN) plasma-line $\left(\mathrm{f}_{\text {radar }}+\mathrm{f}_{\text {heater }}\right)$ observations (Hughes et al., 2003). Simultaneously, optical measurements were made by different filtered camera systems, ranging from all-sky to telescopic, and a photometer. Here we report only on the all-sky camera and photometer optical observations for 630 and $557.7 \mathrm{~nm}$, and the Kodiak centreline $\left(\mathrm{f}_{\text {radar }}\right)$ observations.

The electron gyro-harmonic frequency is given by $f_{\text {gyro }}=\frac{B e}{2 \pi m}$, where $e$ and $m$ are the charge and mass of an electron, respectively, and $B$ is the ionospheric magnetic field strength, which varies with altitude. $B$ was determined from the IGRF-2000 model. In a steady state ionosphere the gyro-harmonic may be found by frequency stepping the pump wave (Kosch et al., 2002a). However, in a weakening ionosphere, which is typical soon after sunset, passing through the gyro-harmonic is more likely to be successful if a constant pump frequency just below the gyro-harmonic is chosen. As the ionosphere weakens, the critical frequency drops but generally the pump wave reflection altitude also increases. This automatically reduces the gyro-harmonic frequency due to the magnetic field strength weakening with increasing altitude. In a correctly designed experiment, the pump frequency becomes greater than the gyro-harmonic before it exceeds the ionospheric critical frequency. In this way, a single frequency sweep through the gyro-harmonic is possible for a non-steady state ionosphere. The upper-hybrid resonance altitude occurs where pump frequency $\left(f_{\text {pump }}\right)$ equals the upper-hybrid frequency $\left(f_{u h r}\right)$. By definition, $f_{u h r}$ corresponds to the altitude where the plasma frequency $\left(f_{\text {plasma }}\right)$ satisfies $f_{\text {plasma }}^{2}=f_{\text {uhr }}^{2}-f_{\text {gyro }}^{2}$ or $f_{\text {plasma }}^{2}=f_{\text {pump }}^{2}-f_{\text {gyro }}^{2}$. Since $f_{\text {pump }}$ and $f_{\text {gyro }}$ are known, the altitude of $f_{\text {plasma }}$ can be determined from the electron density profile in the ionosphere, for which the co-located Digisonde data was used. The upper-hybrid resonance altitude is typically $3-8 \mathrm{~km}$ below the pump wave reflection altitude (Kosch, 2002a). The Digisonde produces time of flight data for echoes over a wide range of frequencies (e.g. typically $1-10 \mathrm{MHz}$ ), which may be converted into an equivalent electron density altitude profile by compensating for the variable propagation speed as a function of density. These data were analysed using both the ARTIST and POLAN algorithms, which produced consistent results.

From 04:02:30 to 04:45:00 UT HAARP was operated at $4.25 \mathrm{MHz}$ in O-mode, corresponding to the third gyroharmonic at $233.5 \mathrm{~km}$. A $150 \mathrm{~s}$ on, $150 \mathrm{~s}$ off cycle was used to prevent interference with the Digisonde whose data are essential to the analysis. The beam was pointed into the local magnetic zenith $\left(204^{\circ}\right.$ azimuth, $75^{\circ}$ elevation), where the maximum optical effect is expected (Kosch et al., 2000; Gurevich et al., 2002; Pedersen et al., 2003; Rietveld et al., 2003). The effective radiated power (ERP) was $47.8 \mathrm{MW}$ with a $-3 \mathrm{~dB}$ beam size of $20.8 \times 29.1^{\circ}$ (geomagnetic $\mathrm{N}$ -

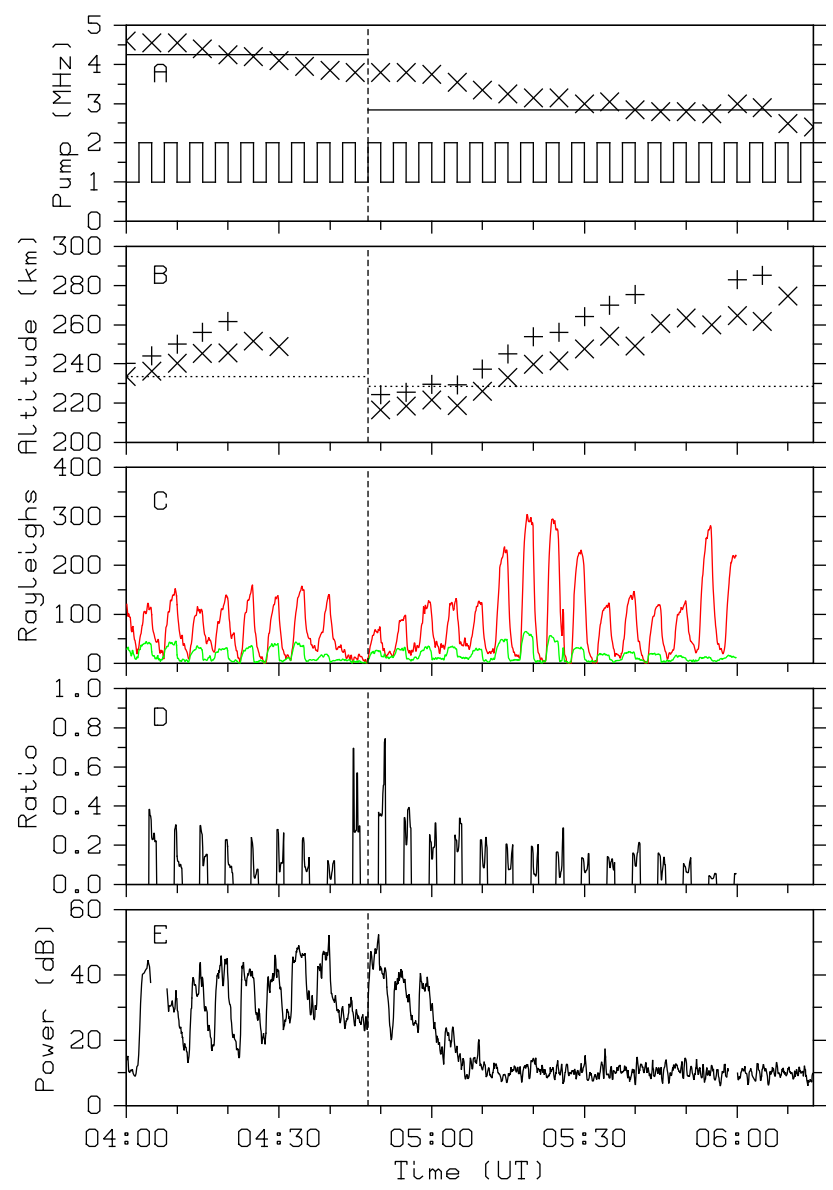

Fig. 1. Results from 25 February 2004. The vertical dashed line in all panels separates the third (left) and second (right) gyro-harmonic parts of the experiment. Panel A shows the pump frequency used (solid lines), ionospheric critical frequency $(x)$ and pump cycle (square waves). Panel B shows the altitude of the respective gyroharmonics (dotted lines), the pump wave reflection altitude measured by the Digisonde $(+)$, and the upper-hybrid resonance altitude $(x)$. Panel $C$ shows the calibrated optical emission enhancement above background for 630 (red line) and 557.7 (green line) nm. Panel D shows the ratio of I(557.7)/I(630). Panel E shows the Kodiak radar backscatter power at $10 \mathrm{MHz}$

$\mathrm{S} \times \mathrm{E}-\mathrm{W})$. From 04:47:30 to 07:00 UT the pump frequency was set to $2.84 \mathrm{MHz}$, corresponding to the second gyroharmonic at $228.5 \mathrm{~km}$, with the same pump cycle and beam pointing direction. The ERP was $10.9 \mathrm{MW}$ with a $-3 \mathrm{~dB}$ beam size of $31.9 \times 44.6^{\circ}$. For the entire experiment, the photometer was pointed parallel to the HAARP beam and has an $8 \times 14^{\circ}$ field of view covering the central part of the pump beam.

Figure 1 shows the results obtained. The vertical dashed line in all panels separates the third (left) and second (right) gyro-harmonic parts of the experiment. Panel A shows the pump frequency used (solid lines), ionospheric critical frequency $(x)$ and pump cycle (square waves). As expected after sunset, the ionospheric critical frequency descended with time, dropping below the pump frequency during each phase 
of the experiment. Panel B shows the altitude of the respective gyro-harmonics (dotted lines), the pump wave reflection altitude measured by the Digisonde (+), and the upperhybrid resonance altitude $(x)$. Unfortunately, the third gyroharmonic was never found, this being due the uncertainty associated with the real time Digisonde analysis. However, the upper-hybrid resonance altitude did pass through the second gyro-harmonic. The data shown in panel B have been manually analysed and have an altitude uncertainty of $\pm 10 \mathrm{~km}$. In addition, the Digisonde echoes come predominantly from overhead whereas the HAARP beam is pointed into the magnetic zenith for which refraction lowers the effective reflection altitude. Ray tracing of the pump wave (not shown) shows that the lowering of the reflection altitude is $<10 \mathrm{~km}$, which is within the measurement uncertainty, and the upperhybrid resonance altitude is still reached everywhere within the pump beam. Hence, no correction has been undertaken for this effect. Issues surrounding the reflection altitude uncertainty are discussed later in detail. After 04:20 UT for the first part of the experiment and 05:40 UT for the second part, the interesting situation arises that the pump wave does not always reflect $(+)$ but still finds the upper-hybrid resonance altitude $(x)$. This situation occurs when the pump frequency is just above the ionospheric critical frequency as shown in panel A.

Panel C of Fig. 1 shows the background-subtracted photometer optical data calibrated in Rayleighs. The enhancements above background for 630 (red line) and 557.7 (green line) $\mathrm{nm}$, synchronised with the pump on cycles, are obvious. For the first part of the experiment (third gyroharmonic) optical emissions continue for the pump-on periods at 04:22:30 and 04:27:30 UT where upper-hybrid resonance is still possible but the Digisonde predicts no reflection can take place. The loss of reflection whilst still finding the upper-hybrid resonance altitude provides additional evidence that upper-hybrid turbulence is the primary mechanism of electron acceleration (Kosch et al., 2002a). Langmuir turbulence is a competing mechanism (Kosch et al., 2004), which occurs only close to the reflection altitude, and is not possible in this case. For the next two pump-on cycles (04:32:30 and 04:37:30 UT) optical enhancements are still clearly seen although the Digisonde predicts that no interaction can take place. This discrepancy may be due to the different beam sizes and pointing directions of the HAARP and Digisonde facilities. Optical emissions are produced for the pump frequency up to $400 \mathrm{kHz}$ above the ionospheric critical frequency (04:37:30 UT). For the pump-on cycle at 04:42:30 UT, no optical emission is produced and the pump frequency is $450 \mathrm{kHz}$ above the critical frequency. This is consistent with observations by Pedersen et al. (2003) who found that photon production cut off sharply for pump frequencies $>500 \mathrm{kHz}$ above the critical frequency. In this situation the pump wave does not reflect, providing evidence that upper-hybrid waves are important for accelerating the electrons (Mishin et al., 2004, 2005).

For the second part of the experiment (second gyroharmonic), optical enhancements (Fig. 1c) up to 300
Rayleighs for $630 \mathrm{~nm}$ and 60 Rayleighs for $557.7 \mathrm{~nm}$ occur for the pump cycles between 05:12:30 and 05:32:30 UT. Both emissions are exceptionally bright considering the modest ERP of 10.9 MW but are consistent with previous observations close to the second gyro-harmonic (Haslett and Megill, 1974). For comparison, a similar red line brightness was achieved at EISCAT using an ERP of $550 \mathrm{MW}$ at $5.423 \mathrm{MHz}$ (Kosch et al., 2004). The brightest optical enhancements occur immediately after the pump frequency passed through the second gyro-harmonic at the upper-hybrid resonance altitude (05:12:30 to 05:32:30 UT). This corresponds to the pump frequency being above the second gyro-harmonic by $\sim 7 \mathrm{kHz}$ for $05: 20$ to $05: 25$ UT. Nothing obviously different happens to the optical signals right on the second gyro-harmonic compared to below it. The enhanced optical emissions near the second gyro-harmonic is opposite to that observed at the third (Kosch et al., 2002a) and fourth (Gustavsson, B., private communication) gyroharmonics where the optical emissions were almost extinguished. The optical enhancement asymmetry about the gyro-harmonic is consistent with the theory of cylindrical upper-hybrid waves trapped in striations (Istomin and Leyser, 2003). After 05:40 UT, optical enhancements continue even though the pump wave penetrates the ionosphere, as discussed before. At 05:55 UT there is another significant enhancement in optical emission brightness at $630 \mathrm{~nm}$, which is clearly not gyro-harmonic related. Unfortunately, the photometer recording terminated at 06:00 UT due to a programming error but the camera data show that these bright enhancements continue until 06:15 UT. We associate this phenomenon with the pump frequency being just above the ionospheric critical frequency. The enhanced optical emission may be due to upper-hybrid resonance occurring over an extended height range giving a brighter column emission. The extended height range is due to the very low gradient in plasma density, which occurs near the F-region peak. This effect has been reported from the mid-latitude SURA ionospheric pump facility (Bernhardt et al., 2000). The height range would normally increase as the ionospheric critical frequency drops quite independently of any chosen pump frequency, which is supported by the ionograms (not shown). This may explain why a similar enhancement was not seen at the end of the first part of the experiment (performed at $4.25 \mathrm{MHz}$ ) as opposed to the second part (performed at $2.84 \mathrm{MHz}$ ).

Figure 2 shows example images of the calibrated artificial optical emissions at $630 \mathrm{~nm}$ from the all-sky imager with the HAARP beam overlaid. The top two panels show images from pumping close to the second gyro-harmonic (05:20 and 05:25 UT). An optical blob is seen centred within the pump beam and on the local magnetic zenith. The optical blob is significantly smaller than the pump beam size, consistent with previous observations (Kosch et al., 2000; Pedersen et al., 2003; Rietveld et al; 2003). The bottom two panels show images (05:50 and 05:55 UT) from pumping just above the ionospheric critical frequency $(\sim 4-9 \mathrm{kHz})$. In these examples irregular optical structures are seen. This is probably due 
to the pump beam illuminating large-scale plasma density irregularities. Enhanced density patches would correspond to regions of optical emissions with the dark areas corresponding to no interaction with the propagating pump wave. It is these patchy structures that correspond to the significantly enhanced $630 \mathrm{~nm}$ optical emissions at the end of the photometer data (see Fig. 1c). A similar effect has been observed in the E-layer at low latitudes (Kagan et al., 2000).

Panel D of Fig. 1 shows the ratio of I(557.7)/I(630). This ratio forms a simple indicator of the relative electron energy with the caveat that pump-induced turbulent plasma is unlikely to have a Maxwellian distribution (Gustavsson et al., 2002). Since the $630 \mathrm{~nm}$ emission has a growth/decay efolding time of $\sim 30 \mathrm{~s}$ in the lower F-region (Gustavsson et al., 2001), the ratio is performed only for the last $90 \mathrm{~s}$ of each pump on period. The apparent amplitude reduction with time is due to the fact that collisional quenching of the $630 \mathrm{~nm}$ emission decreases as the altitude increases (see Fig. 1b) giving a brighter $630 \mathrm{~nm}$ optical emission relative to $557.7 \mathrm{~nm}$. Ignoring the pump pulse just before the change in pump frequency, the ratio falls in the range $\sim 0.05-0.4$, strongly suggesting that a non-Maxwellian electron energy distribution must exist within the heated plasma (Gustavsson et al., 2002). The ratio remains fairly constant $(\sim 0.15-$ 0.2 ) for the period 05:12:30 to 05:32:30 UT when the optical emissions were significantly enhanced for pumping just above the second gyro-harmonic. This indicates that the electron acceleration mechanism probably did not change significantly. The increased accelerated electron flux, as witnessed by the brighter optical emissions, indicates that the acceleration mechanism is more efficient when pumping just above the second gyro-harmonic. This suggests that the striations are enhanced when pumping near the second gyro-harmonic, consistent with Fialer's (1974) observations, which is consistent with the theories by Das and Fejer (1979) and Grach (1979).

Panel E of Fig. 1 shows the Kodiak radar backscatter power over HAARP at $10 \mathrm{MHz}$, which is clearly modulated by the pump cycle. For the first part of the experiment (third gyro-harmonic) all pump cycles produce backscatter, except for the last one. For the pump cycle at 04:42:30 UT virtually no detectable Kodiak backscatter is produced, which corresponds exactly with the lack of optical data (Fig. 1c). Again, this is entirely consistent with upper-hybrid waves being the primary mechanism for producing pump-induced artificial optical emissions (Kosch et al., 2002a,b).

After 05:00 UT, during the second part of the experiment (second gyro-harmonic) the backscatter power decreases to the noise level and remains there (Fig. 1e). This occurred just before the pump wave passed through the second gyroharmonic and is unexpected. Loss of backscatter is ambiguous in that it can either be due to a lack of scatterers (i.e. no striations) or unfavourable propagation conditions resulting in the backscattered wave not reaching the radar receiver. To guarantee radar backscatter, the ray must be very close to orthogonal to the scatterer $\left(<3^{\circ}\right)$ (Fialer, 1974; Senior et al., 2004). Figure 3 shows the intervals of the ray paths which
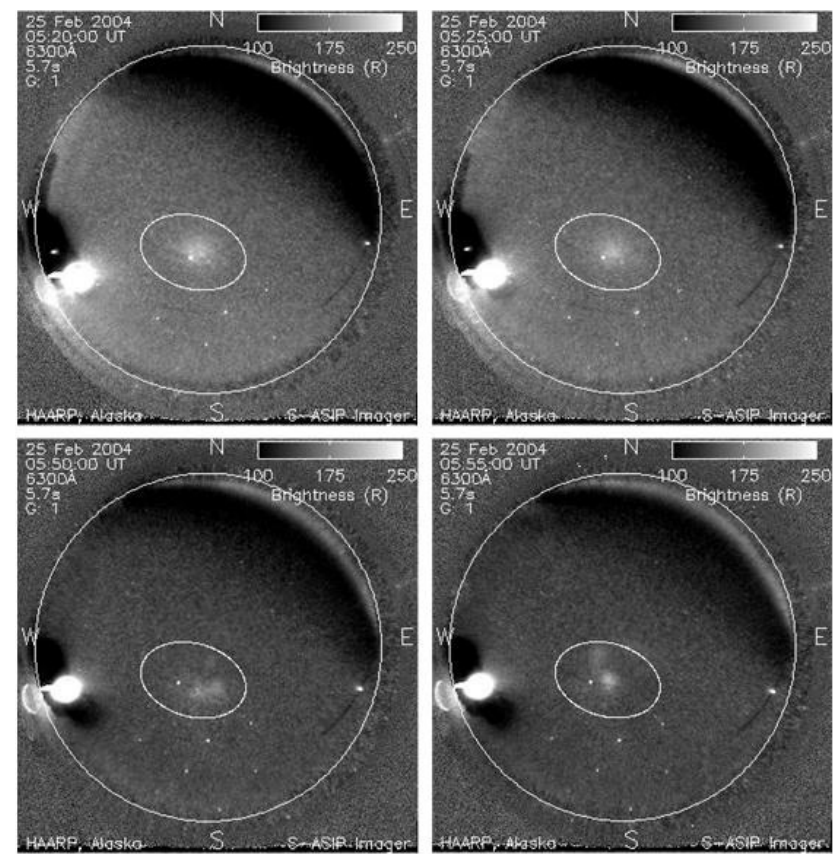

Fig. 2. Example images of the calibrated artificial optical emissions at $630 \mathrm{~nm}$ from the HAARP all-sky imager. The large circle is the horizon. The smaller oval denotes the HAARP beam $-3 \mathrm{~dB}$ locus. The bright white disk near the south-west horizon is the moon. The faint arc near the north-east horizon is a natural aurora. The white spots correspond to stars. The top two panels show images from pumping close to the second gyro-harmonic. The bottom two panels show images from pumping just above the ionospheric critical frequency.

lie within $1^{\circ}$ of orthogonality to the magnetic field line direction over HAARP for the Kodiak radar beam at $10 \mathrm{MHz}$ as a function of range and altitude. All ray paths for Kodiak launch angles from 6 to $30^{\circ}$ in steps of $1^{\circ}$, corresponding to the radar beam pattern, were tested. The ray tracing was performed using the Digisonde data and IGRF-2000 model. HAARP's position is denoted by the vertical line. Black, red, green and blue correspond to 04:20, 04:55, 05:10 and 05:25 UT, respectively. The HAARP beam was dipped $15^{\circ}$ off-vertical and has a half-angle of $\sim 16^{\circ}$ in this direction, so the vertical line closely corresponds to the poleward edge of the beam. At 04:20 (black) and 04:55 (red) UT backscatter is clearly possible over HAARP and does occur (see Fig. 1e). At 05:10 UT conditions for orthogonality become marginal over HAARP and this corresponds to the rapid decline in Kodiak backscatter power. At 05:25 UT, Kodiak backscatter is essentially impossible, corresponding to the noise data in Fig. 1e. Hence, the loss of Kodiak backscatter data after 05:10 UT is easily understood. The loss of suitable propagation conditions for backscatter means that we have no information regarding the strength of the striations, and by association the upper-hybrid waves, around the second gyroharmonic. In all likelihood, striations were being produced by HAARP around the second gyro-harmonic (Fialer (1974), Minkoff et al. (1974)). Not only did Fialer (1974) observe 


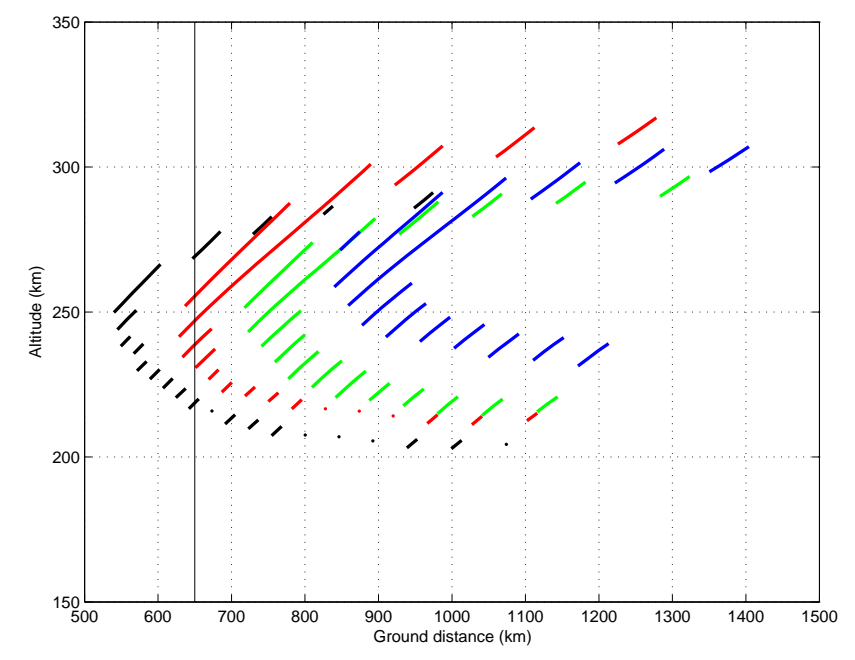

Fig. 3. The intervals of the ray paths within $1^{\circ}$ of orthogonality to the magnetic field line direction over HAARP are shown for the Kodiak radar beam at $10 \mathrm{MHz}$ as a function of range and altitude. HAARP's position is denoted by the vertical line. Black, red, green and blue correspond to 04:20, 04:55, 05:10 and 05:25 UT, respectively, on 25 February 2004.

enhanced striations near the second gyro-harmonic (pump frequency $=2.85 \mathrm{MHz}$ ), but also found evidence that the enhancement maximised when the pump frequency was above the second gyro-harmonic. The loss of Kodiak data during our experiment resulted from a temporary technical limitation in that there was no real time data display when operating the Kodiak radar in the plasma-line mode. This explains why no corrective action (i.e. moving to a lower radar frequency) was taken. Plasma-line data were obtained but are not analysed here.

A $\pm 10 \mathrm{~km}$ change in the pump wave reflection altitude (Fig. 1b), corresponding to the uncertainty in analysing the Digisonde data, results in a similar uncertainty in the upperhybrid resonance altitude. A $10 \mathrm{~km}$ change in either direction has important consequences for the interpretation of the results. However, we are confident that our analysis is correct for the following reasons. If the altitude of the data points in Fig. $1 \mathrm{~b}$ were lowered by $10 \mathrm{~km}$, then the optical enhancement at the second gyro-harmonic would have no asymmetry. In this case, the upper-hybrid resonance altitude would also have passed through the third gyro-harmonic. This would have resulted in a significant reduction in Kodiak backscatter power and optical intensity (Kosch et al., 2002a) prior to 04:10 UT. These effects were not observed (see Figs. 1c and 1e). If the altitude of the data points in Fig. $1 \mathrm{~b}$ were raised by $10 \mathrm{~km}$, then the entire period 04:47:30 to 05:05 UT would have corresponded to the second gyro-harmonic and the optical enhancement asymmetry would be even more pronounced. We conclude that our Digisonde analysis is correct and a small change in altitude $(<10 \mathrm{~km})$ does not affect the results. As mentioned earlier, the Digisonde data comes predominantly from vertically overhead whereas the pump beam was pointed into the magnetic zenith $\left(15^{\circ}\right.$ off vertical $)$.
Refraction lowers the reflection altitude by $<10 \mathrm{~km}$, which is within the measurement uncertainty. Also, by the argument given above, a small reduction in reflection altitude does not affect the conclusions derived from this experiment.

To summarize, pumping just above the second gyroharmonic has the opposite effect to pumping on higher gyroharmonics, namely, there is an enhancement in the artificial optical emissions. This is consistent with the optical observations by Haslett and Megill (1974) and enhanced striations observed by Fialer (1974), and is expected theoretically (Das and Fejer, 1979; Grach, 1979). The maximum optical enhancement occurs when the pump frequency is $\sim 7 \mathrm{kHz}$ above the second gyro-harmonic, consistent with Grach's (1979) theoretical prediction that varying the pump frequency by $1.5-2 \%$ would make the effect disappear. Hence, we provide the first optical experimental evidence of an asymmetry about the gyro-harmonics, with brighter optical enhancements for a pump frequency slightly above the second gyro-harmonic, which was theoretically predicted by Istomin and Leyser (2003). The enhanced optical emissions, and their asymmetry about the gyro-harmonic, are consistent with radar backscatter observations by Fialer (1974). Unfortunately, we have no radar data to corroborate this.

\section{Conclusions}

Artificial optical emissions in the ionospheric F-layer have been produced at the HAARP facility whilst passing through the second gyro-harmonic frequency. A strong enhancement in the 630 and $557.7 \mathrm{~nm}$ emissions was observed, which is opposite to observations at higher gyro-harmonics. Theory predicts a lowering of the threshold for the thermal parametric instability in a plasma where the magnetic field and density gradients are anti-parallel, as is the case in the F-region. However, it is not clear whether this will automatically lead to an enhancement of the striations or greater acceleration of the electrons.

The ratio $\mathrm{I}(557.7) / \mathrm{I}(630)$ remains fairly constant around the second gyro-harmonic frequency, indicating that the electron acceleration mechanism has not changed significantly. The increased accelerated electron flux, as witnessed by the optical data, indicates that the acceleration mechanism is more efficient just above the second gyro-harmonic. This suggests that the striations are enhanced when pumping near the second gyro-harmonic, although we have no direct evidence for this.

The optical enhancement maximised for a pump frequency slightly above $(\sim 7 \mathrm{kHz})$ the second gyro-harmonic. The theory of cylindrical upper-hybrid oscillations trapped within striations predicts an asymmetry of the optical signature about a gyro-harmonic frequency, although the second gyroharmonic was not specifically dealt with. We provide the first optical experimental evidence for this effect.

Artificial optical emissions are produced for pump frequencies above (up to $\sim 400 \mathrm{kHz}$ ) the nominal ionospheric critical frequency. In this situation the pump wave does 
not reflect, providing evidence that upper-hybrid waves are important for accelerating the electrons. Spatially irregular enhanced optical emissions were observed, which may be due to upper-hybrid resonance occurring over an extended height range giving a brighter column emission. The extended height range is due to the very low gradient in plasma density, which occurs near the F-region peak. The patchy structure of the optical emissions is probably due to the "imaging" of large-scale plasma density irregularities in the F-layer.

Acknowledgements. The HAARP program is a Department of Defense project managed jointly by the U.S. Air Force and US Navy. Support from ONR under grant N00014-03-C-0482 is gratefully acknowledged by F. T. Djuth. Support from ONR under grant N00014-03-1-0978 is gratefully acknowledged by E. Gerken.

Topical Editor W. Kofmann thanks two referees for their help in evaluating this paper.

\section{References}

Bernhardt, P. A., Wong, M., Huba, J. D., Fejer, B. G., Wagner, L. S., Goldstein, J. A., Selcher, C. A., Frolov, V. L., and Sergeev, E. N.: Optical remote sensing of the thermosphere with HF pumped artificial airglow, J. Geophys. Res., 105, 10 657-10 671, 2000.

Bond, G. E., Robinson, T. R., Eglitis, P., Wright, D. M., Stocker, A. J., Rietveld, M. T., and Jones, T. B.: Spatial observations by the CUTLASS coherent scatter radar of ionospheric modification by high power radio waves, Ann. Geophys., 15, 1412-1421, 1997, SRef-ID: 1432-0576/ag/1997-15-1412.

Brändström, B. U. E., Leyser, T. B., Steen, A., Rietveld, M. T., Gustavsson, B., Aso, T., and Ejiri, M.: Unambiguous evidence of HF pump-enhanced airglow at auroral latitudes, Geophys. Res. Lett., 26, 3561-3564, 1999.

Das, A. C. and Fejer, J. A.: Resonance instability of small-scale field-aligned irregularities, J. Geophys. Res., 84, 6701-6704, 1979.

Dysthe, K. B., Mjølhus, E., Pecseli, H., and Rypdal, K.: Thermal cavitons, Physica Scripta, T2/2, 548-559, 1982.

Fejer, J. A.: Ionospheric modification and parametric instabilities, Rev. Geophys. Space Phys., 17, 135-153, 1979.

Fialer, P. A.: Field-aligned scattering from a heated region of the ionosphere - Observations at HF and VHF, Radio Sci., 9, 923940, 1974.

Frey, A.: The observation of HF-enhanced plasma waves with the EISCAT/UHF-radar in the presence of strong Landau-damping, Geophys. Res. Lett., 13, 438-441, 1986.

Frolov, V. L., Erukhimov, L. M., Metelev, S. A., and Sergeev, E. N.: Temporal behaviour of artificial small-scale ionospheric irregularities: review of experimental results, J. Atmos. Sol.-Terr. Phys., 18, 2317-2333, 1997.

Frolov, V. L., Sergeev, E. N., Ermakova, E. N., Komrakov, G. P., and Stubbe, P.: Spectral features of stimulated electromagnetic emission, measured in the $4.3-9.5 \mathrm{MHz}$ pump wave frequency range, Geophys. Res. Lett., 28, 3103-3106, 2001.

Grach, S. M.: Thermal parametric instability in ionospheric plasma at frequencies close to $\omega_{H e}$ and $2 \omega_{H e}$, Radiophysics and Quantum Electronics, 22, 357-363, 1979.

Gurevich, A. V.: Nonlinear phenomena in the ionosphere, SpringerVerlag, 1978.
Gurevich, A. V., Zybin, K. P., Carlson, H. C., and Pedersen, T.: Magnetic zenith effect in ionospheric modifications, Phys. Lett. A, 305, 264-274, 2002.

Gustavsson, B., Sergienko, T., Rietveld, M. T., Honary, F., Steen, A., Brändström, B. U. E., Leyser, T. B., Aruliah, A. L., Aso, T., Ejiri, M., and Marple, S.: First tomographic estimate of volume distribution of HF-pump enhanced airglow emission, J. Geophys. Res., 106, 29 105-29 123, 2001.

Gustavsson, B., Brändström, B. U. E., Steen, A., Sergienko, T., Leyser, T. B., Rietveld, M. T., Aso, T., and Ejiri, M.: Nearly simultaneous images of HF-pump enhanced airglow at $6300 \mathrm{~A}$ and 5577 A, Geophys. Res. Lett., 29, doi:10.1029/2002GL015350, 2002.

Haslett, J. C. and Megill, L. R.: A model of the enhanced airglow excited by RF radiation, Radio Sci., 9, 1005-1019, 1974.

Honary, F., Stocker, A. J., Robinson, T. R., Jones, T. B., and Stubbe, P.: Ionospheric plasma response to $\mathrm{HF}$ radio waves operating at frequencies close to the third harmonic of the electron gyrofrequency, J. Geophys. Res., 100, 21 489-21 501, 1995.

Honary, F., Robinson, T. R., Wright, D. M., Stocker, A. J., Rietveld, M. T., and McCrea, I.: First direct observations of the reduced striations at pump frequencies close to the electron gyroharmonics, Ann. Geophys., 17, 1235-1238, 1999,

SRef-ID: 1432-0576/ag/1999-17-1235.

Hughes, J. M., Bristow, W. A., Parries, R. T., and Lundell, E.: SuperDARN observations of ionospheric heater-induced upper hybrid waves, Geophys. Res. Lett., doi:10.1029/2003GL018772, 2003.

Istomin, Ya. N. and Leyser, T. B.: Electron acceleration by cylindrical upper hybrid oscillations trapped in density irregularities in the ionosphere, Phys. Plasmas, 10, 2962-2970, 2003.

Kagan, L. M., Kelley, M. C., Garcia, F., Bernhardt, P. A., Djuth, F. T., Sulzer, M. P., and Tepley, C. A.: The structure of electromagnetic wave-induced 557.7-nm emission associated with a sporadic-E event over Arecibo, Phys. Rev. Lett., 85, 218-221, 2000.

Kelley, E. C., Arce, T. L., Salowey, J., Sulzer, M., Armstrong, W. T., Carter, M., and Duncan, L.: Density depletions at the 10-m scale induced by the Arecibo heater, J. Geophys. Res., 100, 17367$17376,1995$.

Kosch, M. J., Rietveld, M. T., Hagfors, T., and Leyser, T. B.: Highlatitude HF-induced airglow displaced equatorwards of the pump beam, Geophys. Res. Lett., 27, 2817-2820, 2000.

Kosch, M. J., Rietveld, M. T., Kavanagh, A. J., Davis, C., Yeoman, T., Honary, F., and Hagfors, T.: High-latitude pump-induced optical emissions for frequencies close to the third electron gyroharmonic, Geophys. Res. Lett., 29, 2112-2115, 2002a.

Kosch, M. J., Rietveld, M. T., Yeoman, T. K., Cierpka, K., and Hagfors, T.: The high-latitude artificial aurora of 21 February 1999: An analysis, Adv. Polar Upper Atmos. Res., 16, 1-12, 2002 b.

Kosch, M. J., Rietveld, M. T., Senior, A., McCrea, I. W., Kavanagh, A. J., Isham, B., and Honary, F.: Novel artificial optical annular structures in the high latitude ionosphere, Geophys. Res. Lett., 31, L12805, doi:10.1029/2004GL019713, 2004.

Leyser, T. B., Gustavsson, B., Brändström, B. U. E., Steen, A., Honary, F., Rietveld, M. T., Aso, T., and Ejiri, M.: Simultaneous measurements of high-frequency pump-enhanced airglow and ionospheric temperatures at auroral latitudes, Adv. Polar Upper Atmos. Res., 14, 1-11, 2000.

Leyser, T. B.: Stimulated electromagnetic emissions by highfrequency electromagnetic pumping of the ionospheric plasma, 
Space Sci. Rev., 98, 223-328, 2001.

Minkoff, J., Kugelman, P., and Weissman, J.: Radio frequency scattering from a heated ionospheric volume, 1, VHF/UHF fieldaligned and plasma line backscatter measurements, Radio Sci., 9, 941-955, 1974.

Mishin, E. V., W. J. Burke and T. Pedersen, On the onset of HF-induced airglow at HAARP, J. Geophys. Res., 109, doi: 10.1029/2003JA010205, 2004.

Mishin, E. V., Burke, W. J., and Pedersen, T.: HF-induced airglow at magnetic zenith: theoretical considerations, Ann. Geophys., 23, 47-53, 2005,

\section{SRef-ID: 1432-0576/ag/2005-23-47.}

Mjølhus, E.: On linear conversion in magnetized plasma, Radio Sci., 6, 1321-1339, 1990.

Pedersen, T. R. and Carlson, H. C.: First observations of HF heaterproduced airglow at the High Frequency Active Auroral Research facility: Thermal excitation and spatial structuring, Radio Sci., 36, 1013-1026, 2001.

Pedersen, T. R., McCarrick, M., Gerken, E., Selcher, C., Sentman, D., Carlson, H. C., and Gurevich, A.: Magnetic zenith enhancement of HF radio-induced airglow production at HAARP, Geophys. Res. Lett., 30, doi:10.1029/2002GL016096, 2003.

Ponomarenko, P. V., Leyser, T. B., and Thidé, B.: New electron gyroharmonic effects in the HF scatter from pump-excited magnetic field-aligned ionospheric irregularities, J. Geophys. Res., 104, 10 081-10 087, 1999.

Rietveld, M. T., Kosch, M. J., Blagoveshchenskaya, N. R., Kornienko, V. A., Leyser, T. B., and Yeoman, T. K.: Ionospheric electron heating, optical emissions and striations induced by powerful HF radio waves at high latitudes: Aspect angle dependence, J. Geophys. Res., 108, 1141, doi:10.1029/2002JA009543, 2003.

Robinson, T. R.: The heating of the high latitude ionosphere by high power radio waves, Physics Reports, 179, 79-209, 1989.
Robinson, T. R., Honary, F., Stocker, A. J., Jones, T. B., and Stubbe, P.: First EISCAT observations of the modification of F-region electron temperatures during RF heating at harmonics of the electron gyro frequency, J. Atmos. Terr. Phys., 58, 3385-3395, 1996.

Robinson, P. A.: Nonlinear wave collapse and strong turbulence, Rev. Modern Phys., 69, 507-573, 1997.

Robinson, T. R., Stocker, A., Bond, G., Eglitis, P., Wright, D., Jones, T. B., and Rietveld, M. T.: First CUTLASS-EISCAT heating results, Adv. Space Res., 21(5), 663-666, 1998.

Senior, A., Borisov, N. D., Kosch, M. J., Yeoman, T. K., Honary, F., and Rietveld, M. T.: Multi-frequency HF radar measurements of artificial F-region field-aligned irregularities, Ann. Geophys., 22, 3503-3511, 2004,

SRef-ID: 1432-0576/ag/2004-22-3503.

Sipler, D. P. and Biondi, M. A.: Measurements of $\mathrm{O}\left({ }^{1} \mathrm{D}\right)$ quenching rate in the F region, J. Geophys. Res., 77, 6202-6212, 1972.

Stocker, A. J., Honary, F., Robinson, T. R., Jones, T. B., Stubbe, P. and Kopka, H.: EISCAT observations of large scale electron temperature and electron density perturbations caused by high power HF radio waves, J. Atmos. Terr. Phys., 54, 1555-1572, 1992.

Stocker, A. J., Honary, F., Robinson, T. R., and Jones, T. B.: Anomalous absorption during artificial modification at harmonics of the electron gyrofrequency, J. Geophys. Res., 98, 13627$13634,1993$.

Stubbe, P., Stocker, A. J., Honary, F., Robinson, T. R., and Jones, T. B.: Stimulated electromagnetic emissions and anomalous HF wave absorption near electron gyroharmonics, J. Geophys. Res., 99, 6233-6246, 1994.

Stubbe, P.: Review of ionospheric modification experiments at Troms $\varnothing$, J. Atmos. Terr. Phys., 58, 349-368, 1996. 